\title{
AIP
}

\section{Impact of plasma noise on a direct thrust measurement system}

S. J. Pottinger, D. Lamprou, A. K. Knoll, and V. J. Lappas

Citation: Rev. Sci. Instrum. 83, 033504 (2012); doi: 10.1063/1.3692740

View online: http://dx.doi.org/10.1063/1.3692740

View Table of Contents: http://rsi.aip.org/resource/1/RSINAK/v83/i3

Published by the AIP Publishing LLC.

\section{Additional information on Rev. Sci. Instrum.}

Journal Homepage: http://rsi.aip.org

Journal Information: http://rsi.aip.org/about/about_the_journal

Top downloads: http://rsi.aip.org/features/most_downloaded

Information for Authors: http://rsi.aip.org/authors

\section{ADVERTISEMENT}

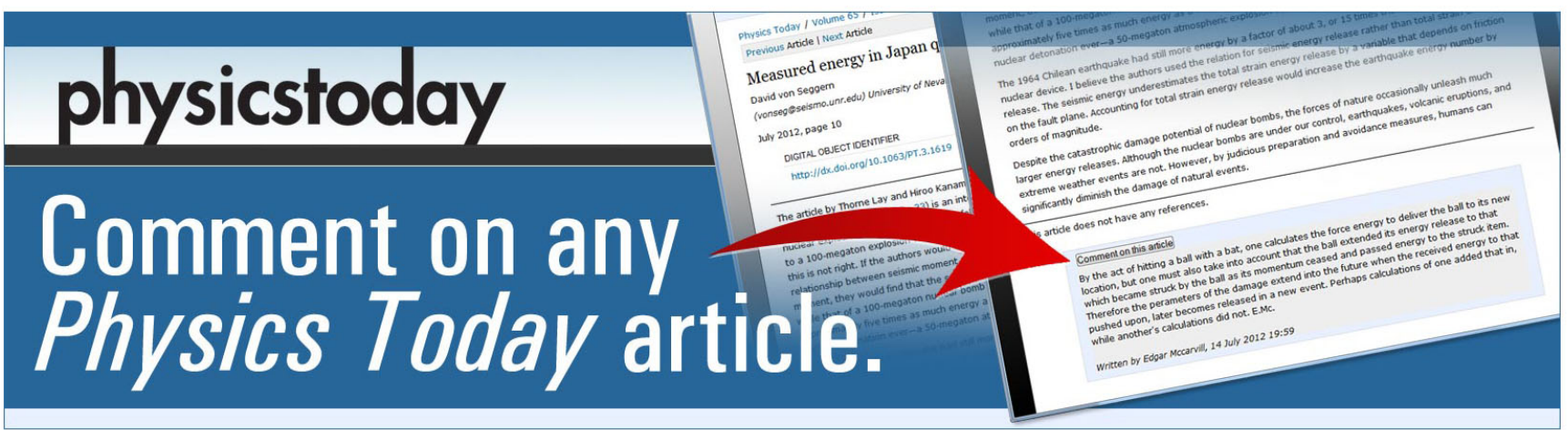




\title{
Impact of plasma noise on a direct thrust measurement system
}

\author{
S. J. Pottinger, D. Lamprou, A. K. Knoll, and V. J. Lappas \\ Surrey Space Centre, University of Surrey, Guildford GU2 7XH, United Kingdom
}

(Received 20 June 2011; accepted 19 February 2012; published online 16 March 2012)

\begin{abstract}
In order to evaluate the accuracy and sensitivity of a pendulum-type thrust measurement system, a linear variable differential transformer (LVDT) and a laser optical displacement sensor have been used simultaneously to determine the displacement resulting from an applied thrust. The LVDT sensor uses an analog interface, whereas the laser sensor uses a digital interface to communicate the displacement readings to the data acquisition equipment. The data collected by both sensors show good agreement for static mass calibrations and validation with a cold gas thruster. However, the data obtained using the LVDT deviate significantly from that of the laser sensor when operating two varieties of plasma thrusters: a radio frequency (RF) driven plasma thruster, and a DC powered plasma thruster. Results establish that even with appropriate shielding and signal filtering the LVDT sensor is subject to plasma noise and radio frequency interactions which result in anomalous thrust readings. Experimental data show that the thrust determined using the LVDT system in a direct current plasma environment and a RF discharge is approximately a factor of three higher than the thrust values obtained using a laser sensor system for the operating conditions investigated. These findings are of significance to the electric propulsion community as LVDT sensors are often utilized in thrust measurement systems and accurate thrust measurement and the reproducibility of thrust data is key to analyzing thruster performance. Methods are proposed to evaluate system susceptibility to plasma noise and an effective filtering scheme presented for DC discharges. () 2012 American Institute of Physics. [http://dx.doi.org/10.1063/1.3692740]
\end{abstract}

\section{INTRODUCTION}

Electromagnetic noise is inherent to virtually all plasma technologies owing to the rich and complex wave phenomenon fundamental to these devices. The interaction of this plasma noise on diagnostic sensors is of particular concern because the development of electric thrusters is reliant on accurate high precision measurements to assess the plasma characteristics and thrust generated by the engines. Direct thrust measurement is a tool that is widely used to characterize thrust levels ranging from micro Newtons up to Newtons for both pulsed and steady state discharges. There are two thrust measurement systems typically used for the characterization of electric propulsion technologies: the torsion balance and pendulum thrust stand. The torsion balance is used routinely to determine the thrust produced by pulsed discharges ${ }^{1-3}$ and has also been used for steady state systems. ${ }^{4,5}$ Its mechanical structure allows a rapid response to an applied impulse and thrusts of the order of nano Newtons have been measured to $\pm 2 \%$ error; $^{6}$ however, a damping system is required which adds to the system complexity. The pendulum design thrust stand employed in the current investigation consists of a simple mechanical design that provides easy interchangeable mounting for various thrusters. A maximum load of $15 \mathrm{~kg}$ may be supported and a measurement uncertainty of $\pm 3 \%$ of the measured value is achieved with the use of a laser system. A detailed description of the thrust stand is provided in Sec. III.

Thrust stands rely on high precision displacement measurements. The sensors employed for these measurements include the linear variable differential transformer (LVDT), laser interferometry, ${ }^{2}$ laser displacement sensors, fiber optic linear displacement sensors, ${ }^{5}$ and strain gauges. ${ }^{7}$ The LVDT has been widely used for thrust characterization due to cost, simplicity, and accuracy. ${ }^{1,3,6,8-10}$ The current investigation has verified that LVDTs are susceptible to electromagnetic interference and radio frequency (RF) interactions when testing both a laboratory DC and RF powered plasma thruster. A thorough treatment of the subject of plasma noise and its impact on sensor systems is required to establish a means of minimizing the impact of noise on thrust measurement data. Performance characterizations of electric propulsion systems often overlook this aspect or apply post-processing methods such as Fourier and wavelet transforms ${ }^{8}$ or subtract the power spectrum of the plasma discharge from the data signal. ${ }^{5}$ Publications have demonstrated that with a judicial choice of power connections, placement of the RF power supply and ensuring that the ratio of forward power to reflected power is maximized, RF noise may be minimized. ${ }^{11}$ However, the current investigation has shown that even when these countermeasures are employed, the interaction of plasma noise on the LVDT sensor can be significant, see Sec. V B. The findings of the current investigation indicate that digital laser sensor systems offer an increased robustness and reliability compared to analog LVDTs without the need for external filtering of output signals and extensive shielding methods. This paper provides a detailed account of methods that may be employed to assess the susceptibility of a thrust measurement system to electromagnetic interference caused by plasma noise. For thrust stands and sensors that have not been calibrated to meet industry standards (i.e., systems that have been developed in house by researchers), the results demonstrate that it is best practice to first evaluate the response of the system under 
conditions where zero thrust should be registered. The thrust stand should be bolted to prevent movement or the thruster should be mounted perpendicular to the axis of thrust stand motion. This approach provides an indication of the filtering required. Sections II-IV describe the thrusters used to validate the thrust measurement systems and the calibration processes undertaken to ensure the accuracy of processed data. The experimental results obtained from a LVDT and laser optical displacement sensor are compared.

\section{THRUSTER CONCEPTS}

\section{A. Hollow cathode thruster}

The hollow cathode thruster (HCT) is a conventional hollow cathode used in a stand-alone configuration to generate thrust. Hollow cathodes are an established technology that have demonstrated reliable performance in excess of $28000 \mathrm{~h}$ for applications including the main electron source for electron bombardment ion thrusters, external neutralizers, and plasma contactors, they also act to sustain the plasma discharge of Hall effect thrusters. ${ }^{12,13}$ It was suggested in the $1970 \mathrm{~s}^{14}$ that HCs can produce stand-alone thrust and more recently experimental results have demonstrated thrust levels up to $2.5 \mathrm{mN}$ using an indirect thrust measurement system. ${ }^{15}$ For the case of the HCT evaluated in this investigation (see Fig. 1) an insert impregnated with earth metal oxides is heated to $\sim 1100^{\circ} \mathrm{C}$ which results in electron emission. The liberated electrons interact with the propellant gas generating ions and a discharge is initiated between the cathode and anode (keeper electrode). A detailed understanding of the fundamental physics which drives the operation of the device remains an ongoing area of investigation and further work is required to establish a viable thrust generation mechanism. Theories have been put forward to suggest that the thrust is produced by electrothermal acceleration of high temperature neutrals or electrostatic acceleration of ions through a potential gradient between the cathode and keeper electrode or anode. ${ }^{16,17}$ It has also been suggested that HCs have the capability to produce high energy ions which may contribute to

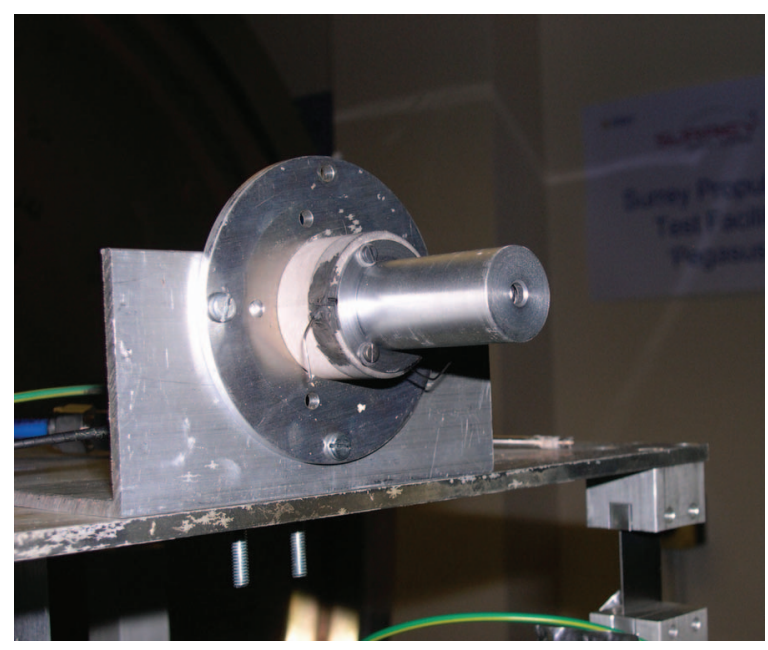

FIG. 1. HCT mounted on thrust stand. the thrust generated by the device..$^{18}$ Although a comprehensive theory for thrust generation mechanisms has yet to be firmly established, the primary focus of the work presented is to measure the thrust produced by a HCT by means of direct thrust measurement and assess the interaction of the plasma noise generated by this device on the LVDT and laser sensor systems. It should be noted that HCs operate in distinct modes where the extreme cases are referred to as the spot mode (a low noise discharge) and plume mode which generates high levels of electrical noise relative to the spot mode. ${ }^{18}$ The results presented correspond to the HCT operating in the spot mode.

\section{B. Helicon double layer thruster}

The helicon double layer thruster (HDLT) is a RF driven plasma thruster that accelerates ions across a double layer in order to generate a propulsive force. The thruster is described in detail in a number of references ${ }^{19-22}$ and its main components are shown in Fig. 2. Key factors that dictate thruster performance are the properties of the helicon discharge $e^{23}$ and the strength of the double layer. The plasma discharge of the HDLT is generated via the deposition of $13.56 \mathrm{MHz} \mathrm{RF}$ power through a copper antenna. This antenna is connected to an impedance matching network which ensures effective power coupling from the RF generator to the plasma. Once the helicon discharge is formed, a double layer develops spontaneously in the region of the source tube exit in the presence of an imposed diverging magnetic field. The double layer forms within a limited pressure range that is dependent on the choice of operating propellant. ${ }^{24}$ The double layer is a finite region with dimensions of less than three centimeters depth corresponding to a potential drop of the order of tens of volts ${ }^{25}$ resulting in ion acceleration in a collimated macroscopically neutral beam. The presence of the double layer replaces the two or three grid systems typically used in ion engines for ion acceleration. As a result, the lifetime issues associated with grid degradation are eliminated. The external beam is neutralized by trapped electrons with insufficient energy to

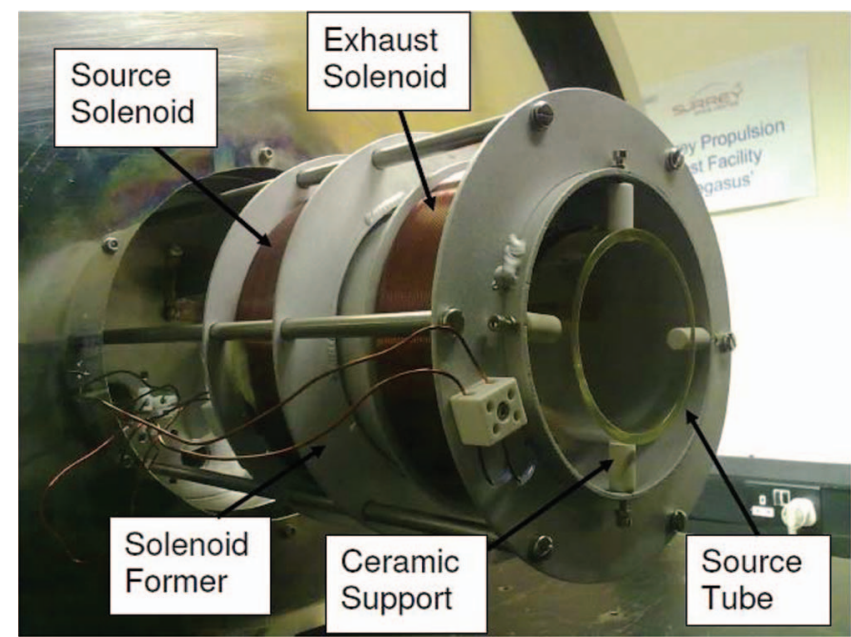

FIG. 2. HDLT mounted in the vacuum chamber. 


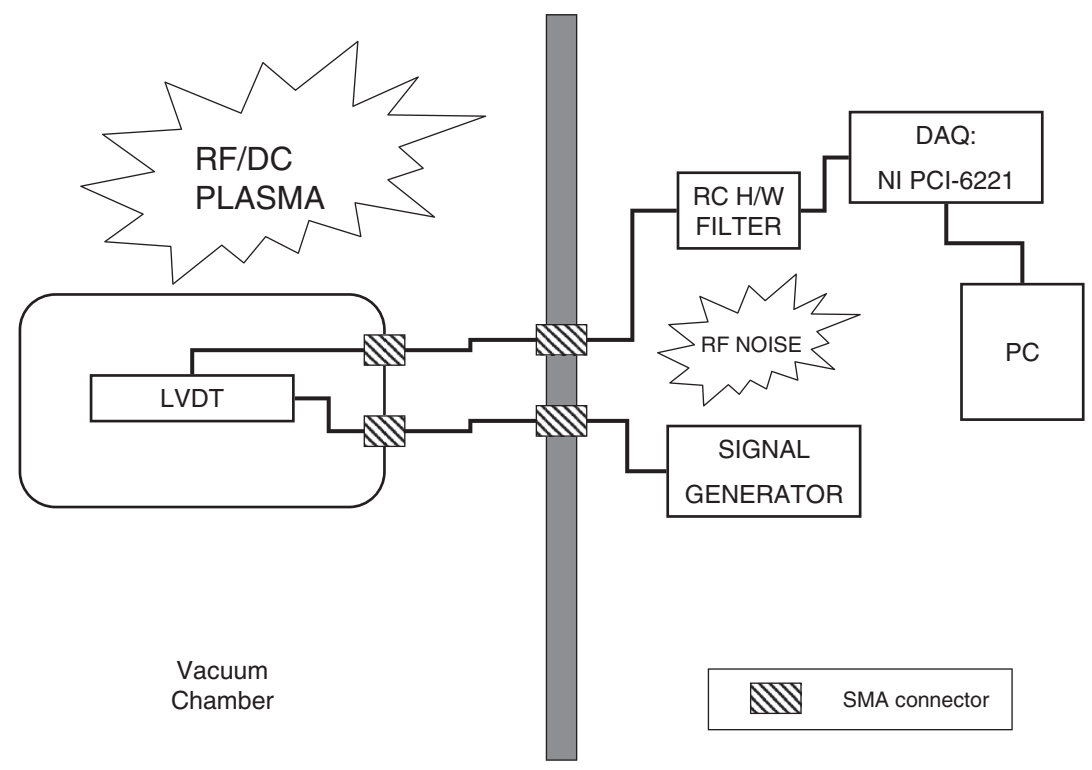

FIG. 3. LVDT block diagram.

overcome the low potential side of the double layer and travel upstream. Therefore, a neutralizing cathode is not required and the complexity of the thruster is reduced. The HDLT is a comparatively new propulsion system compared to the established electric propulsion technologies. Sections III-V outline initial efforts to characterize the thrust of a non-optimized laboratory prototype and the issues encountered related to the interaction of plasma noise on direct thrust measurements.

\section{THRUST MEASUREMENT SYSTEM}

A custom designed thrust stand was developed that could be operated as a simple pendulum or as an inverted pendulum. In each configuration the thrust is measured by comparing the deflection caused by the thrust with the deflection caused by the application of a known calibration mass. The mechanical design of the thrust stand consists of a supporting table and flextures connected to supporting struts. The rigidity of the flextures dictates the degree of deflection of the thrust stand and is subject to the mass of the thruster undergoing characterization. The pendulum configuration is used to evaluate the thrust produced by the HDLT. The propellant feed line is mechanically decoupled from the system and electrical harnessing to the thruster are flexible coils which minimize the impact of system damping on the sensitivity of the deflection measurement. The inverted pendulum design is used to characterized HCT performance. The impact of the propellant feed line stiffness is mitigated by the use of a flexible feed line and the electrical harnessing is again coiled to reduce friction. The linear response in the $0-10 \mathrm{mN}$ and $0-4 \mathrm{mN}$ range for the pendulum and inverse pendulum configurations are shown in Sec. IV.

The displacement of the thrust stand under an applied force has been evaluated using two independent sensors, a LVDT and laser sensor. The LVDT sensor supplied by Macro Sensors (model PR-812-200) has a measuring range of $5 \mathrm{~mm}$ with a sensitivity of $285 \mathrm{mVmm}^{-1}$. The input signal to the LVDT is provided by a laboratory signal generator that supplies an ac signal of $3 \mathrm{Vrms}$ at $3 \mathrm{kHz}$. The LVDT sensor is encapsulated in an electrostatically shielding aluminum box with SMA input and output connectors. The shielding box also provides non-contact access for the LVDT core. Shielded cables are used throughout the setup with isolated SMA feedthroughs on the vacuum chamber flange as shown in Fig. 3. Grounding of the LVDT and the LVDT box is performed via the SMA cable shielding outside the vacuum chamber to avoid ground loops, see Fig. 4. The output from the LVDT is an analogue signal that is passed through a hardware RC (resistor-capacitor) filter with a corner frequency of $4 \mathrm{kHz}$. The signal is sampled at $250 \mathrm{k}$ samples per second by a NI PCI-6221 data acquisition card. A software implemented $20 \mathrm{~Hz}$ Butterworth bandpass filter centred at the LVDT input frequency calculates the rms of the voltage $\left(V_{\text {rms }}\right)$ signal and outputs the average rms voltage for a period of $1 \mathrm{~s}$. The data presented below include both the unfiltered and filtered results for the HCT, taken before and after the software bandpass filter, respectively. The data shown for the HDLT include unfiltered data only (see Sec. V B).

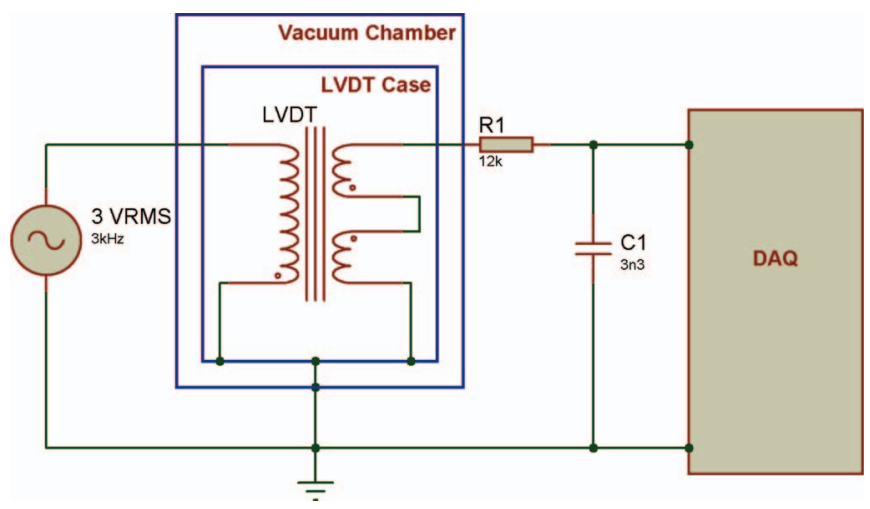

FIG. 4. LVDT circuit diagram 
A Micro-Epsilon laser optical displacement sensor, model number ILD 1700-50, has been mounted directly below the LVDT box for the experimental trials discussed in Secs. IV-V. The setup is composed of a semiconductor laser producing a light beam with wavelength $\lambda=670 \mathrm{~nm}$ that is reflected from the central strut of the thrust stand. The displacement is determined via triangulation within the sensor unit. A measuring rate of $312.5 \mathrm{~Hz}$ with 3 point averaging and a resolution of $3 \mu \mathrm{m}$ has been adopted for all experiments. The ILD 1700-50 has an internal ADC (analogue to digital converter) and RS422 interface.

The two sensor systems were operated simultaneously to evaluate the capability of the setup to provide accurate thrust measurements for direct current electric thrusters and radio frequency ion engines. Initial tests were performed to confirm that the response of the thrust stand was linear over the thrust range of interest. These experiments demonstrate a good agreement between the outputs of the two sensor systems. The response of the thrust measurement system to a cold gas thruster was then evaluated before operating the system in a plasma environment. The thrust stand is operated within a vacuum chamber with dimensions of $1.2 \mathrm{~m}$ length and $1.2 \mathrm{~m}$ diameter for all experiments. The vacuum system achieves a background pressure of $\sim 1 \times 10^{-6}$ mbar without load, during thruster operation a minimum pressure of $\sim 2 \times 10^{-4}$ mbar is achieved.

\section{CALIBRATION}

\section{A. Static mass characterization}

The thrust stand has been designed to facilitate the use of an in situ calibration method using a static mass. A $9.5 \mathrm{~g}$ mass is suspended from the thrust stand and displaced by a remotely operated stepper motor. The stepper motor has a resolution of 200 steps per revolution, and operates in 1/8 step mode at a frequency of $100 \mathrm{~Hz}$. The applied calibration force is calculated for a known displacement using the geometry shown in Fig. 5. The displacement $s$, of the calibration mass

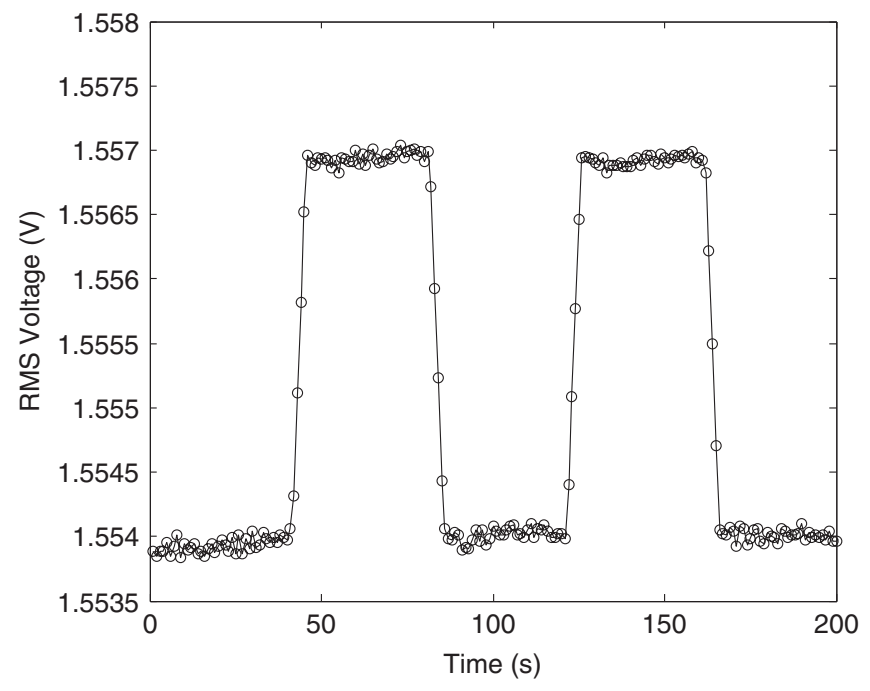

FIG. 6. LVDT sensor calibration curve showing filtered data.

is determined via the number of steps and the known geometry of the pulley system. The force component as a function of displacement and output voltage is determined for the laser sensor and the LVDT respectively. Figures 6 and 7 show representative calibration curves for both sensor systems for an applied force of $6.2 \mathrm{mN}$, in both cases the calibration mass begins in the null position where zero force is applied along the axis of the thrust stand, i.e., the $z$ axis. Force is then applied and removed in time intervals of $40 \mathrm{~s}$. High frequency oscillations are observed for the laser sensor output as a result of background vibrations of the test facility, see Fig. 7 which are not demonstrated by the LVDT system due to a higher sampling rate of $250 \mathrm{kHz}$ compared to a maximum of $0.3125 \mathrm{kHz}$ for the laser system. For the given LVDT sensitivity of $285 \mathrm{mVmm}^{-1}$, the output voltage may be converted to displacement for direct comparison with the output of the laser sensor system. By altering the number of revolutions of the stepper motor/pulley system the calibration force can be

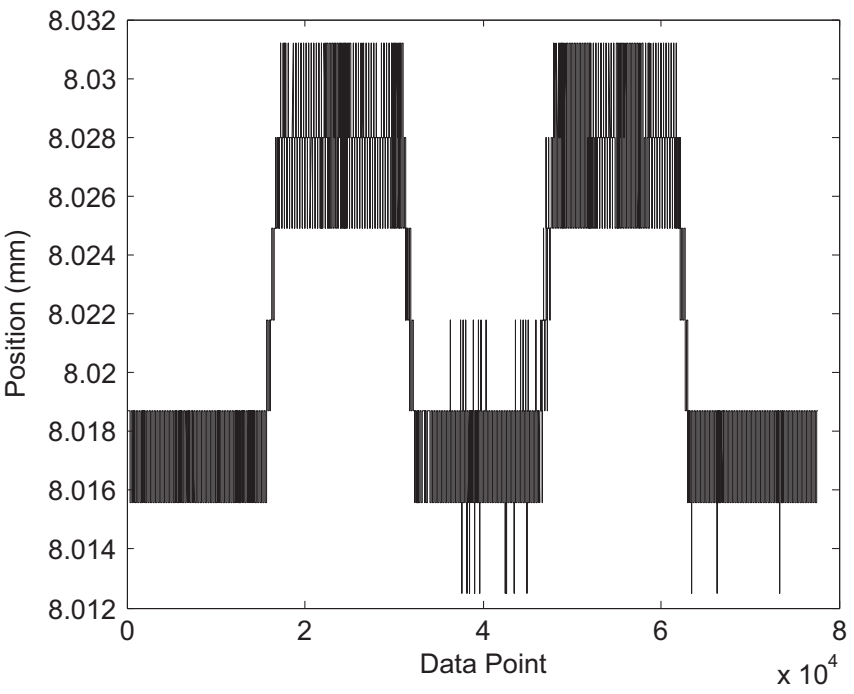

FIG. 5. Geometry of calibration mass and pulley system.

FIG. 7. Laser sensor calibration curve. 


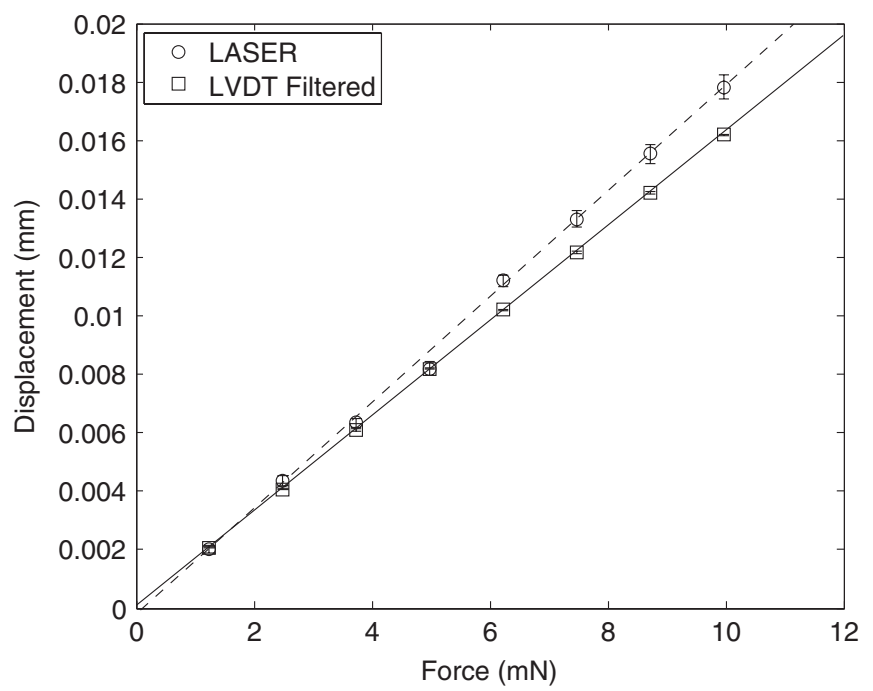

FIG. 8. Pendulum thrust stand response to mass calibration.

adjusted. Two thrust stand configurations were investigated; the pendulum and inverted pendulum. The inverted pendulum configuration provides a greater sensitivity to displacement under an applied force for a given pendulum length. However, the simple pendulum is able to support a greater weight. For this reason, the light weight HCT was characterized on the inverted pendulum, while the heavier HDLT was characterized with the simple pendulum. For the pendulum configuration a range of $1.2 \mathrm{mN}-10.0 \mathrm{mN}$ was investigated, and a $0.8 \mathrm{mN}-4.2 \mathrm{mN}$ force was applied for the inverted pendulum configuration. These upper and lower bounds were tailored to the expected range of thrust produced by the HDLT and HCT devices, respectively. The resulting displacement of the thrust stand in each configuration versus the calibration force is shown in Figs. 8 and 9. Each data point represents the average of 6-10 repeat measurements, and the error bar is the standard uncertainty of the measurement. ${ }^{26,27}$ These results demonstrate that both thrust stand configurations exhibit a linear response with increasing applied force for both sen-

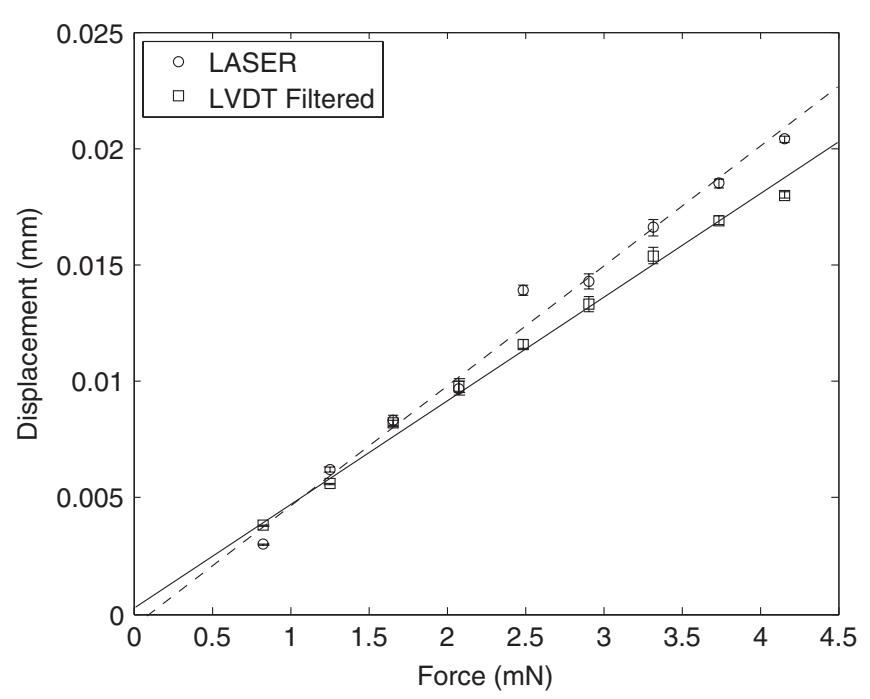

FIG. 9. Inverted pendulum thrust stand response to mass calibration. sor systems. However, there is a discrepancy in the measured displacement between the two sensors, which can most likely be attributed to the conversion of the LVDT signal from voltage to displacement. It is possible that there is a slight difference between the manufacturer recommended conversion factor for voltage to displacement and the actual behavior of the LVDT sensor used in our setup. The second explanation relates to the measurement uncertainty. The laser system has a measurement range of $50 \mathrm{~mm}$ and a manufacturer reported linearity of $40 \mu \mathrm{m}$ and resolution of $3 \mu \mathrm{m}$. The discrepancy between the LVDT and laser measurement sensor falls below the reported resolution of the device. It is important to recognize that, regardless of the cause for the difference in the measured displacements recorded from the two sensors, the absolute value of displacement is not critical in establishing the level of thrust. The thrust level is based on the change in position resulting from thruster firing, which is determined separately for each sensor on the basis of calibration measurements.

\section{B. Cold gas characterization}

A simple cold gas thruster composed of a plenum volume and a 1/4 in. diameter circular orifice was used to characterize both thrust stand configurations. Krypton propellant was used in volumetric flow rates of $10-80 \mathrm{sccm}$ corresponding to $0.6-$ $5.0 \mathrm{mgs}^{-1}$. The propellant flow rate is controlled by an automated mass flow controller rated from 0 to $200 \mathrm{sccm}$. Cold gas calibration results are shown in Figs. 10 and 11. Theoretical curves are shown for the force $F$ produced by a choked flow and free molecular flow for a mass flow rate $\dot{m}$ with an absolute temperature of $T=25^{\circ} \mathrm{C} .{ }^{28}$ The choked flow limit is given by

$$
F=u \dot{m}=\sqrt{\gamma R T} \dot{m},
$$

where $\gamma=\frac{C_{P}}{C_{V}}$ is the ratio of specific heats $(\gamma=1.667$ for noble gases), and $R$ is the specific gas constant ( $R=99.2 \mathrm{Jkg}^{-1} \mathrm{~K}^{-1}$ for krypton propellant). The thrust

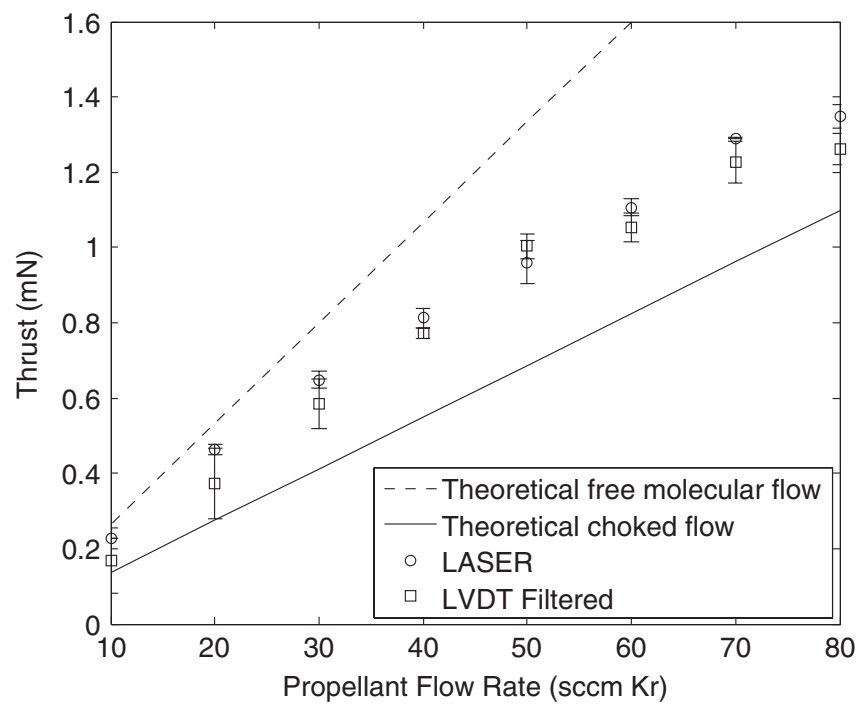

FIG. 10. Pendulum thrust stand response to cold gas thruster. 


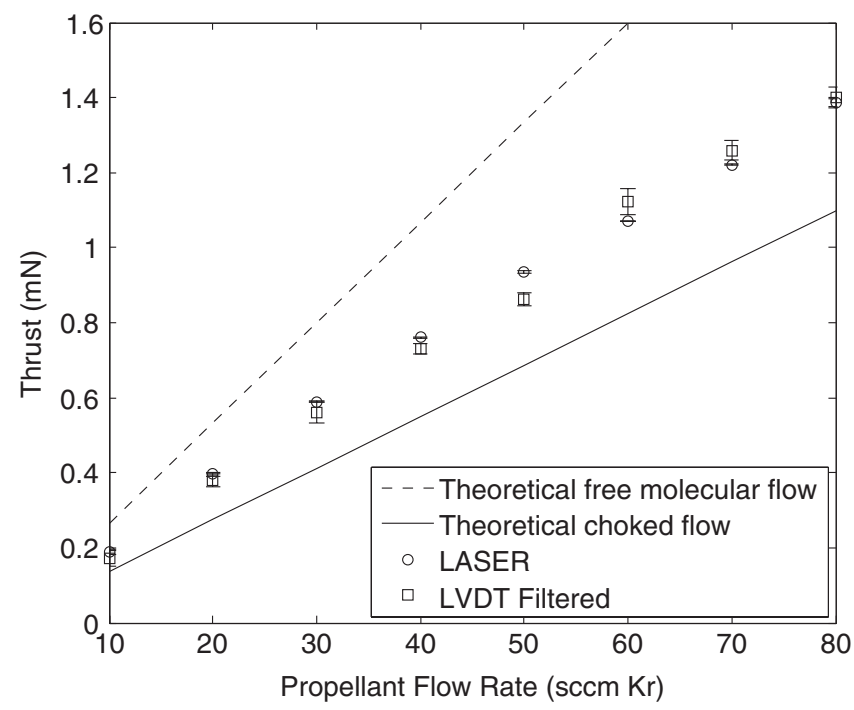

FIG. 11. Inverted pendulum thrust stand response to cold gas thruster.

produced through free molecular flow is given by

$$
F=\sqrt{\frac{2 \pi k T}{M_{A}}} \dot{m},
$$

where $M_{A}$ is the atomic mass and $k$ is Boltzmann's constant.

The thrust stand produces thrust values that show good agreement between the filtered LVDT data and laser sensor. As expected, the measured thrust is linear with flow rate, and lies between the choked flow and free molecular limits. Reasonable agreement is achieved comparing the results obtained by the two thrust stand configurations.

\section{SENSOR PERFORMANCE IN A PLASMA ENVIRONMENT}

\section{A. Hollow cathode thruster}

The data presented in Figs. 12 and 13 have been obtained with a HCT operating with an enclosed keeper configuration

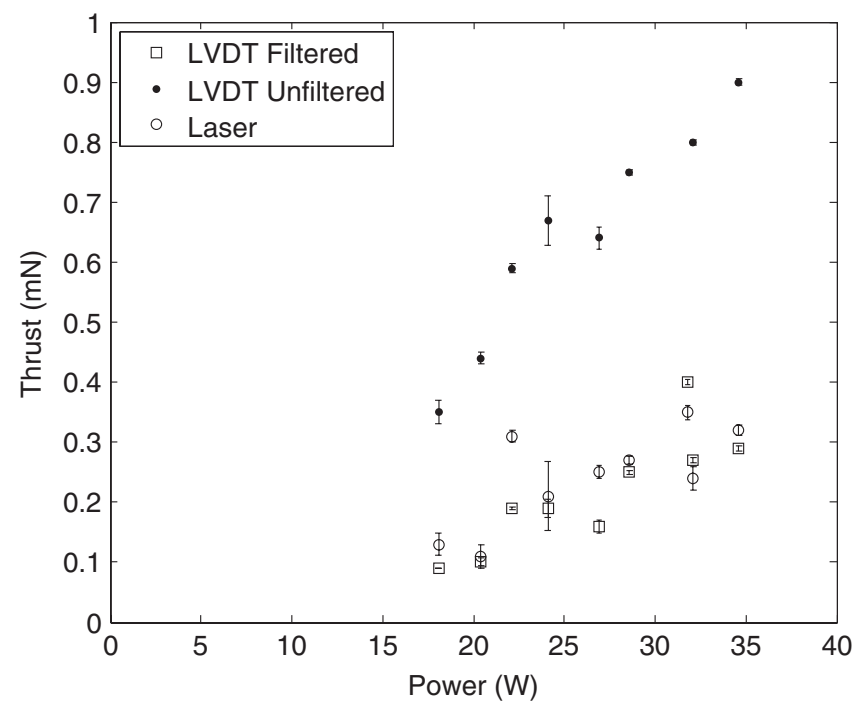

FIG. 12. Thrust as a function of power for the HCT firing forward.

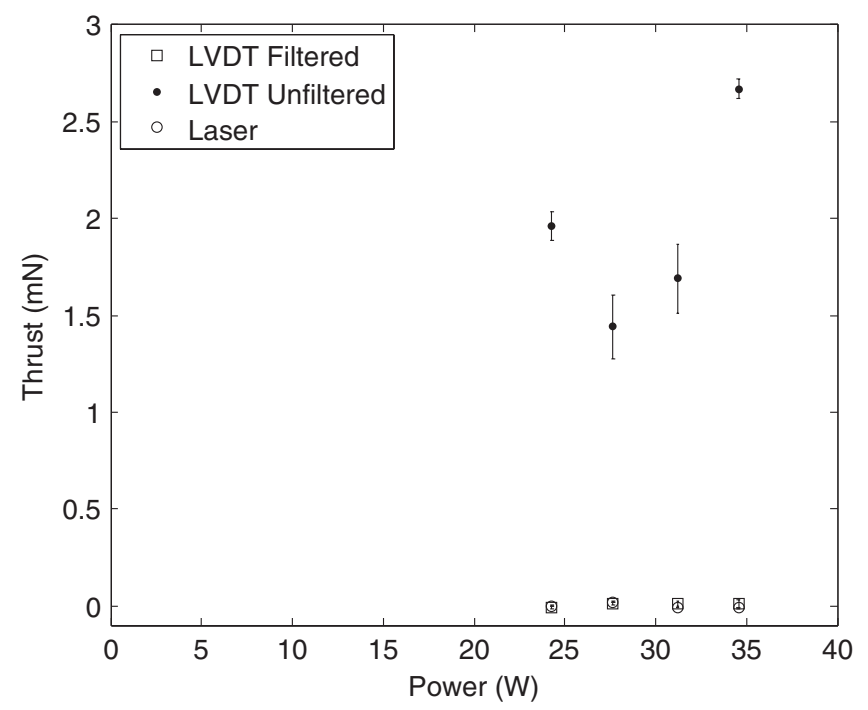

FIG. 13. Thrust as a function of power for the HCT firing at $90^{\circ}$.

(no external anode) with a propellant flow rate of $25 \mathrm{sccm}$ of xenon. In order to demonstrate that the sensor systems were able to function effectively in a DC plasma environment, the HCT was operated in two configurations: (i) the thruster was aligned in the direction of thrust stand displacement, (ii) the thruster was aligned at $90^{\circ}$ to the thrust stand axis. The thrust data presented in Figs. 12 and 13 represent the contribution of the plasma discharge to thrust and is measured by turning the discharge power on and off under conditions of continuous gas flow. There should be zero displacement of the thrust stand in scenario (ii) as the applied force is perpendicular to the displacement axis. The unfiltered LVDT signal produces apparent thrust readings (see Fig. 12) that are significantly elevated compared to the filtered LVDT and laser results. Figure 13 shows an apparent thrust measured by the LVDT for the HCT operating in the $90^{\circ}$ orientation, which is clearly in error. In contrast, the laser displacement measurement behaves as expected, showing no measurable thrust in the $90^{\circ}$ orientation of the HCT. The explanation put forward by this study is that anomalous ac components of the LVDT signal, driven by the interaction of the plasma noise with the LVDT sensor, corrupt the determination of the rms voltage that is used to quantify the displacement. In this case, a software implemented bandpass filter is able to rectify the problem and achieve reasonable agreement between the laser and LVDT measurements.

\section{B. Helicon double layer thruster}

The thrust produced by a HDLT operating with a plasma source tube with an outer diameter of $80 \mathrm{~mm}$, inner diameter of $75 \mathrm{~mm}$, and length of $172 \mathrm{~mm}$ was investigated using the pendulum thrust stand configuration. The diverging magnetic field was supplied by a coaxial solenoid magnet with a peak field strength of $\sim 100 \mathrm{G}$ positioned flush with the channel exit, as shown in Fig. 2. A propellant flow rate of $16 \mathrm{sccm}$ $\left(1 \mathrm{mgs}^{-1}\right)$ of krypton was used throughout testing. Figure 14 shows the thrust produced by the HDLT as measured using 


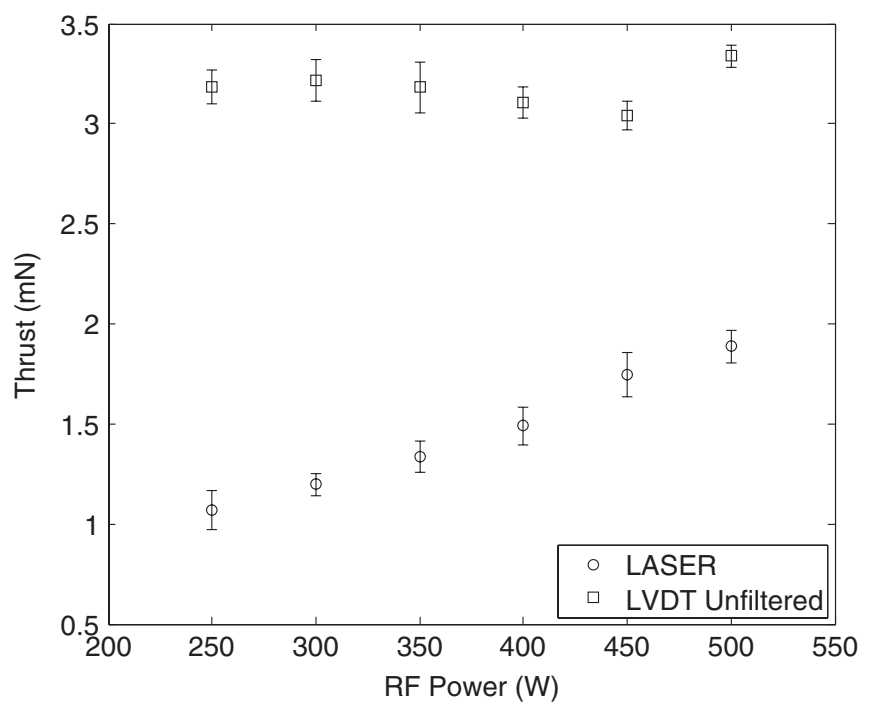

FIG. 14. Thrust as a function of power for the HDLT operating with $\mathrm{Kr}$ propellant, $B_{\text {peak }} \sim 100 \mathrm{G}$.

the laser sensor and unfiltered LVDT signal. The laser data show a linear trend of increasing thrust with increasing RF input power, whereas the LVDT data do not follow the same trend and the measured thrust levels are significantly higher. Applying a Butterworth filter to the LVDT data as was done with the DC discharge proved ineffective in eliminating the impact of plasma and RF interactions on the LVDT sensor. Further investigation is required in order to identify appropriate signal filters for operation with an RF discharge.

\section{CONCLUSIONS}

The performance of two different displacement sensor systems have been evaluated for direct thrust measurements in the presence of a DC and RF powered plasma thruster. Results have shown that the force measured by a digital laser sensor and LVDT show good agreement during the calibration process and for a cold gas thruster. However, when operated in a plasma environment a discrepancy appears between the force measured by the sensors; the LVDT produces anomalous readings of thrust, whereas the digital laser sensor remains unaffected. By combining the use of both hardware and software filters, as well as properly shielding cables and interfaces in the experimental setup, the problems due to the interaction of the plasma noise with the LVDT sensor could be mitigated for a small DC powered thruster (the HCT). However, these measures proved insufficient for the RF powered plasma thruster. This study suggests that while the LVDT sensor is a commonly employed device for direct thrust measurements, it is not the best candidate for thrust measurements of plasma devices. If this technology is to be used in a plasma environment evaluation of the response of the device in various experimental setups and thruster operating conditions should be undertaken in order to assess the degree to which plasma noise impacts the LVDT output signal. The application of appropriate filtering based on the outcomes this assessment may then be employed to counteract the effect of plasma noise.

\section{ACKNOWLEDGMENTS}

This research has been funded through the University of Surrey's strategic partnership agreement with EADS Astrium. The authors would like to thank Matthew Perren and Robert Laine for their support during this project. The authors also thank members of the Australian National University's Space Plasma, Power and Propulsion Group for valuable technical support relating to the HDLT.

${ }^{1}$ T. W. Haag, Rev. Sci. Instrum. 68, 2060 (1997).

${ }^{2}$ E. A. Cubbin, J. K. Ziemer, E. Y. Choueiri, and R. G. Jahn, Rev. Sci. Instrum. 68, 2339 (1997).

${ }^{3}$ H. Koizumi, K. Komurasaki, and Y. Arakawa, Rev. Sci. Instrum. 75, 3185 (2004).

${ }^{4}$ S. Orieux, C. Rossi, and D. Esteve, Rev. Sci. Instrum. 73, 2694 (2002).

${ }^{5}$ M. Gamero-Castano, Rev. Sci. Instrum. 4, 4509 (2003).

${ }^{6}$ A. J. Jamison, A. D. Ketsdever, and E. P. Muntz, Rev. Sci. Instrum. 73, 3629 (2002)

${ }^{7}$ R. J. Stephen, K. Rajanna, V. Dhar, K. G. Kalyan Kumar, and S. Nagabushanam, Meas. Sci. Technol. 12, 1568 (2001).

${ }^{8}$ B. C. D'Souza and A. D. Ketsdever, Air Force Research Laboratory (AFMC), AFRL/PRSA, 10 E. Saturn Blvd., Edwards AFB, CA, Technical Report 93524-7680, 2005.

${ }^{9}$ N. Gascon, W. S. Crawford, R. L. Corey, and M. A. Cappelli, in Proceedings of the 29th International Electric Propulsion Conference, Princeton, NJ, 2005.

${ }^{10}$ K. G. Xu and M. L.R. Walker, Rev. Sci. Instrum. 80, 055103 (2009).

${ }^{11}$ A. W. Kieckhafer and M. L.R. Walker, Rev. Sci. Instrum. 81, 075106 (2010).

${ }^{12}$ A. Sengupta, J. R. Brophy, and K. D. Goodfellow, in Proceedings of the 39th AIAA/ASME/SAE/ASEE Joint Propulsion Conference and Exhibit, Huntsville, AL, 2003.

${ }^{13}$ S. D. Kovaleski, M. J. Patterson, G. C. Soulas, and T. R. Sarver-Verhey, in Proceedings of the 27th International Electric Propulsion Conference, Pasadena, CA, 2001.

${ }^{14}$ A. Snyder and B. A. Banks, NASA Technical Note, NASA TN D-6705, 1972.

${ }^{15}$ A. N. Grubisic, S. B. Gabriel, and D. G. Fearn, in Proceedings of the 30th International Electric Propulsion Conference, Florence, Italy, 2007.

${ }^{16}$ A. N. Grubisic and S. B. Gabriel, in Proceedings of the 31 st International Electric Propulsion Conference, Ann Arbor, MI, 2009.

${ }^{17}$ I. G. Mikellides, I. Katz, D. M. Goebel, and J. E. Polk, J. Appl. Phys. 98, 113303 (2005).

${ }^{18}$ D. M. Goebel, K. K. Jameson, I. Katz, and I. G. Mikellides, Phys. Plasmas 14, 103508 (2007).

${ }^{19}$ M. D. West, C. Charles, and R. W. Boswell, J. Propul. Power 24, 134 (2008).

${ }^{20}$ C. Charles, J. Phys. D 42, 163001 (2009).

${ }^{21}$ C. Charles, R. W. Boswell, and M. A. Lieberman, Appl. Phys. Lett. 89, 261503 (2006).

${ }^{22}$ S. J. Pottinger, T. Harle, V. J. Lappas, W. Cox, T. Lafleur, P. Alexander, C. Charles, R. W. Boswell, M. Perren, and R. Laine, in Proceedings of Space Propulsion, San Sebastian, Spain, 2010.

${ }^{23}$ E. E. Scime, A. M. Keesee, and R. W. Boswell, Phys. Plasmas 15, 058301 (2008).

${ }^{24}$ C. Charles, Plasma Sources Sci. Technol. 16, R1 (2007).

${ }^{25}$ M. A. Lieberman, C. Charles, and R. W. Boswell, J. Phys. D 39, 3294 (2006).

${ }^{26}$ S. Bell, The National Physical Laboratory, Technical Report ISSN 13686550, 2001.

${ }^{27}$ R. Blott, S. Gabriel, and D. Robinson, Technical Report SEP/ESA/21662/OP1/Issue5/2010.

${ }^{28}$ G. P. Sutton and O. Biblarz, Rocket Propulsion Elements, 7th ed. (Wiley, New York, 2001). 